\title{
Coronavirus disease 2019 pandemic and anxiety: a longitudinal study in 287 Brazilians
}

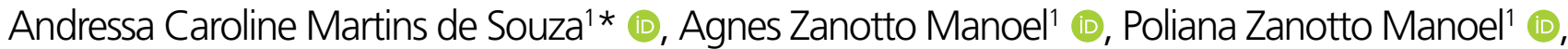

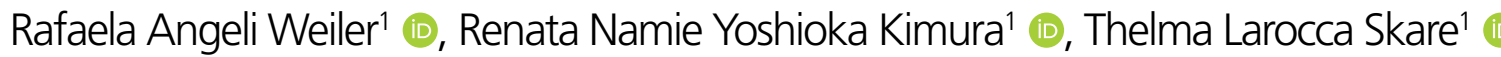

\section{SUMMARY}

OBJECTIVE: The aim was to study the prevalence of symptoms of anxiety and post-traumatic stress in a sample of Brazilians and their relationship with sex, age, and work situation, and compare if these symptoms change with 8 weeks of quarantine.

METHODS: Online survey to collect epidemiological data and apply the Beck Anxiety Inventory (BAI) and Impact of Event Scale-Revised (IES-R). Eight weeks later, the researchers requested the same requirements to complete the BAI and IES-R and compare the results.

RESULTS: The sample of 287 answered the first and second questionnaires, being $72.8 \%$ women, with a median age of 22 years. In the first interview, the median BAI was 12 (7-19) and the median IES-R was 27.0 (15-40); in the second, the median BAI was 11 (6-22) and the IES-R was 30 (15-41) with $p<00001$ and 0.09 , respectively. Anxiety levels were worse in females $(p<0.0001$ for both BAI and IES-R) and in those who worked/studied in the area of health ( $p=0.001$ for BAI and 0.01 for IES-R). There was a negative correlation between age and anxiety ( $p<0.0001$ for BAI and IES-R).

CONCLUSIONS: A high prevalence of anxiety that lowered after 8 weeks were found. Anxiety was worse in females, in younger people, and in those who worked/studied in the area of health.

KEYWORDS: COVID-19. Pandemics. Quarantine. Anxiety.

\section{INTRODUCTION}

In March 2020, the World Health Organization (WHO) recognized the coronavirus disease 2019 (COVID-19) outbreak as pandemic ${ }^{1}$. No drug can be considered efficient against this infection nor has an effective vaccine been developed until now. So, to avoid the infection spreading, social distancing and quarantine have been implemented, bringing segregation, rupture of previous habits, and economical losses.
In this context, additional stress can be triggered by a fear of contamination and anxiety about the loss of one's own life or that of loved ones.

The infections have been identified first in China at the end of 2019, and investigators have found a reduction in positive emotions and life satisfaction and an increased rate of depression, anxiety, and even suicidality in this population during the period of isolation ${ }^{2-4}$.

'Faculdade Evangélica Mackenzie do Paraná - Curitiba (PR), Brazil.

*Corresponding author: dessacmds@hotmail.com

Conflicts of interest: the authors declare there are no conflicts of interest. Funding: none.

Received on December 19, 2020. Accepted on February 07, 2021. 
The same pattern has been observed in other regions. A German study showed generalized anxiety in $44.9 \%$, depression in $14.3 \%$, and psychological distress in $65.2 \%$ of the study sample which were found to be more common in women and younger people ${ }^{5}$. Another study conducted in the USA among people aged from 18 to 30 years showed high levels of depression (43.3\%), high anxiety scores (45.4\%), and high levels of post-traumatic stress disorder (PTSD) symptoms (31.8\%) which were less frequent in Asian Americans than in whites ${ }^{6}$. They also observed that anxiety was less likely to be reported in Hispanics/Latinos ${ }^{6}$. A large Iranian study showed that anxiety was higher among women, among people who followed corona-related news closely, among those in the age group of 21- 40 years and those who have had a relative with the disease ${ }^{7}$. A systematic review by Xiong et al. ${ }^{8}$ corroborated the findings of high levels of anxiety, depression, PTSD, psychological distress, and stress in several countries (i.e., China, Spain, Italy, Iran, USA, Turkey, Nepal, and Denmark), bringing attention to the fact that unemployment, student status, and presence of chronic/psychiatric illnesses should be added to the previously mentioned associated risk factors.

An interesting observation, comparing the physical and mental health of citizens living in countries with an opposite point of views about wearing face masks (China that encouraged face masks and Poland that discouraged them), showed that the infrequent use of face masks was associated with more physical symptoms, more frequent medical consultation, COVID-19 testing, hospitalization, and also deterioration of emotional status. This study highlights the importance of health education not only in preventing the disease from spreading but also in reducing its psychological impact?

Additionally, it was noted that there was an increase in the post-traumatic stress symptoms when quarantine exceeded 10 days $^{10}$.

The health care workers who were quarantined may have more significant symptoms of post-traumatic stress than people in other work areas. The COVID-19 pandemic has placed a significant strain on health care institutions that were required to allocate all the efforts and resources in appropriated protective equipment and minimize non-essential services, and in the front-line health care staff exposed to the disease and the psychological adversities brought by patient's care. Chew et al. ${ }^{11}$, studying 1,146 individuals, observed that health care workers not medically trained were particularly vulnerable to psychological adversities. Besides, health care workers who experienced physical symptoms had worse psychological outcomes than those who did not. Some of the known reasons are fear of spreading the infection to colleagues and family members and the dilemma of applying for sick leave in an already strained area. In this context, the health care worker may develop fear, stigmatization, and feeling of exclusion while displaying the physical indicators of virus infection ${ }^{12}$.

Not only health care workers have been affected by the COVID-19 pandemic but also others in whom the social isolation constrained their jobs and brought income reduction and recession ${ }^{13}$. Two-third of the 1,423 respondents involved in a cross-sectional study from Vietnam reported an important reduction in their quality and quantity of working which was more accentuated among females ${ }^{13}$.

Returning to work during the COVID-19 epidemic is another situation that may be associated with psychological effects. About $10.8 \%$ of responders of 673 questionnaires in China showed PTSD without significant differences between technicians/workers and executive/managers. Anxiety (3.8\%), depression $(3.7 \%)$, stress $(1.5 \%)$, and insomnia $(2.3 \%)$ were similarly detected although at a lower rate. Factors associated with the severity of psychiatric problems were the presence of physical symptoms, poor physical health, considering the return to work as a health hazard, and marital status ${ }^{14}$.

Resilience to stress may suffer from cultural and social influences. Therefore, it may diverge according to the study population. Herein, we studied a sample of Brazilian patients aiming to know the prevalence of anxiety and PTSD and if the persistence of quarantine would change this prevalence.

\section{METHODS}

This study was approved by the local Committee of Ethics in Research under protocol 30725520.7.0000.0103. This is a prospective study based on an Internet questionnaire. Electronic informed consent was obtained before answering the questions. The first questionnaire was applied from March 20 to 30 through Google forms announced on Facebook, Instagram, and WhatsApp groups and was intended for individuals aged 18 years or above. Data collection included epidemiological information, questions on social distancing and quarantine, the Beck Anxiety Inventory (BAI ${ }^{15}$, and Impact of Event Scale-Revised (IES-R) ${ }^{16}$.

The IES-R is a self-application instrument for screening the symptomatology of PTSD which demonstrates good discriminating validity and diagnostic utility and can be used in any stage (acute, chronic, and late) of the development of symptoms. It uses a Likert scale on which an individual answers the questions based on 7 days before the application of the questionnaire. The scale has 22 items distributed across 3 subscales (i.e., avoidance, intrusion, and hyperstimulation) that contain the criteria for evaluating PTSD available in DSM-IV. The score for each question ranges from 0 to 4 points and the calculation 
of the score of each subscale is obtained through the average of the items; the total score is the sum of the scores of the subscales. According to this instrument, PTSD can be classified as follows: 0-23 as absent, $24-32$ as mild, 33-36 as moderate, and $>37$ as severe psychological impact ${ }^{16}$.

IES-R has been used and previously validated to measure post-traumatic disorders symptoms during the COVID-19 pandemic in several countries ${ }^{17-19}$.

The Beck anxiety scale or Beck inventory is a self-report questionnaire with 21 multiple choice questions, which is used to measure the severity of an individual's anxiety. These questions are about how the individual has felt in the previous week and it is expressed as common symptoms of anxiety (such as sweating and feelings of anguish). Each question has four possible answers, and the one that most closely resembles the individual's mental state should be signaled. The instrument has a maximum score of 63 points, and the participants are classified according to the degrees of anxiety: minimum: $0-10$, mild: 11-19, moderate $20-30$, and severe: $31-63^{15}$.

Patients who participated in this survey were contacted again after 8 weeks and invited to answer again the IER-S and Beck inventory to verify if the symptoms have changed with isolation time.

Obtained data were analyzed in tables of frequency and contingency. Data distribution was studied by using the ShapiroWilk test. The Mann-Whitney $U$ test was used to compare two numerical data (i.e., Beck inventory and IES-R according to epidemiological variables). The Wilcoxon test was used to compare two paired numeric data (comparison of two results of Beck inventory and two results of IES-R). The chi-squared test was used to compare the categorical values of Beck and the categorical values of the IES-R between the two evaluations. The correlation of age with Beck inventory and results of IES-R was done by using the Spearman's test. The adopted significance was $5 \%$. Tests were calculated using GraphPad Prism ${ }^{\circledast}$ version 6.01 software.

\section{RESULTS}

1. Results of the first evaluation:

The survey was answered by 287 individuals. Their epidemiological data are shown in Table 1.

In this sample, the mean result of the Beck inventory was 12 (range 0-46; IQR 6-22). According to this instrument, $121 / 267$ (42.1\%) had minimal anxiety, 78/287 (27.1\%) had mild anxiety, 56/287 (19.5\%) had moderate anxiety, and 31/287 $(10.8 \%)$ had severe anxiety.

The median IES-R was 30.0 (range 0-76; IQR 15-41). In 117/287 (40.7\%), PTSD was absent; in 56/287 (19.5\%),
Table 1. Epidemiological profile of the study sample.

\begin{tabular}{l|c} 
Sex - male/female & $78 / 209-27.1 / 72.8 \%$ \\
\hline Median age - years (IQR) & $22.0(20.0-25.0)$ \\
\hline Working/studying in health area (\%) & $96 / 287(33.4)$ \\
\hline Stayed on quarantine (\%) & $266 / 287(92.6)$
\end{tabular}

IQR: interquartile range.

PTSD was mild; in 23/287 (8.0\%), PTSD was moderate, and in $92 / 287$ (32.0\%), PTSD was severe.

According to gender, the comparison of BAI scores and IES-R showed that males had a median value of 7.5 (IQR 3.0-14.0) and females had a median value of 14 (IQR 8.0-25.0) with $\mathrm{p}<0.0001$; the median IES-R in males was 22.0 (IQR 7.0-31.2) and in females it was 32.0 (IQR 18.0-44.0), with $\mathrm{p}<0.0001$.

The comparison of the results of Beck anxiety inventory in those who were working/studying in the health area was 14.0 (IQR 8.0-24.0) and in those who were not working in this area was 10.0 (IQR 4.0-15.0) with $\mathrm{p}=0.001$. The IES-R in those who were related to the health area was 31.0 (IQR 17.0-42.0) and in those who were not related to the health area was 23.0 (13.2-34.0) with $\mathrm{p}=0.01$.

The comparison of $\mathrm{BAI}$ in those who were keeping quarantine had a median value of 13.0 (IQR 7.0-23.0) and in those who were not keeping it had a median value of 6.0 (IQR 2.511.5 ), with $\mathrm{p}=0.0003$. The median IES-R in those who were on quarantine was 30.0 (IQR 17.0-41.0) and in those who were not keeping it was 12.0 (IQR 6.5-24.5), with $p=0.0001$.

A correlation study between age and Beck inventory results showed $\mathrm{p}<0.0001$ (rho $=-0.24 ; 95 \% \mathrm{CI}-0.35$ to -0.12 ). The correlation of age with IES-R also showed $\mathrm{p}<0.0001$ (rho $=-0.22$; $95 \%$ CI -0.33 to -0.10 ).

2. Results of the second evaluation:

During the period between the two evaluations, 239/287 (83.2\%) kept the quarantine, $8 / 287(2.7 \%)$ who were not on quarantine previously entered in isolation, 27/287 (9.4\%) left the quarantine, and 13/287 (4.55) who were not on isolation in the first evaluation continued not keeping isolation.

The comparison of Beck anxiety inventory between the first and second visits is shown in Figure 1 in which the second evaluation showed lower levels.

The comparison of IES-R in the two evaluations is shown in Figure 2.

The variation of scores in the Beck inventory between the second and first evaluation (delta Beck) had a median value of 
A

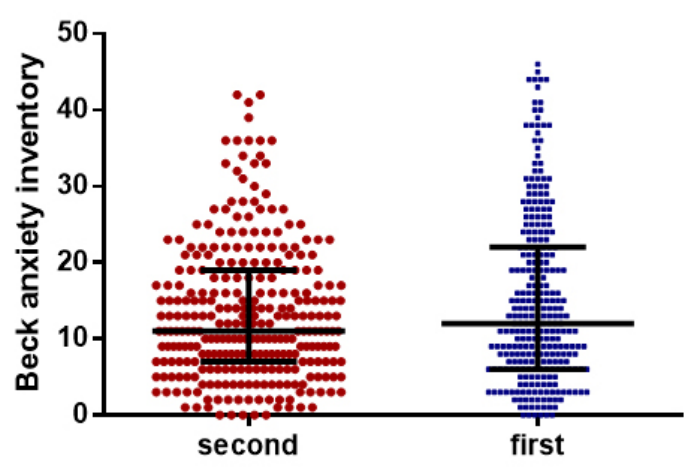

B

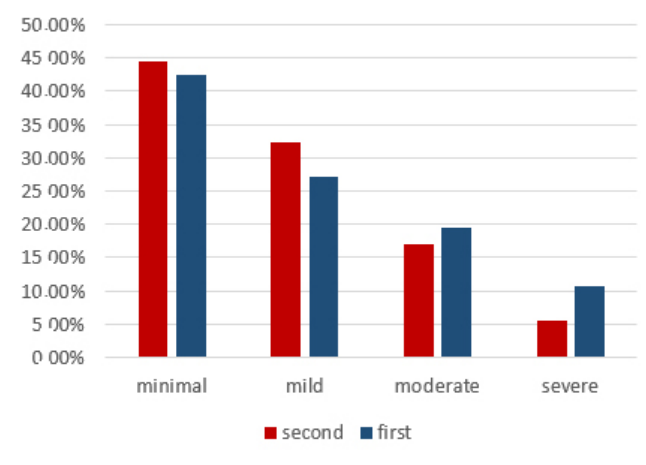

Figure 1. Comparison of Beck anxiety inventory between second and first evaluation. (A) Numeric values: $p<0.0001$; second evaluation: median value of 11.0 (IQR 7-19); first evaluation: median value of 12.0 (IQR 6.0-22.0). (B) Categorical values: $\mathrm{p}=0.08$; second evaluation: minimal $=44.5 \%$, mild $=32.4 \%$, moderate $=17.0 \%$, and severe $=5.5 \%$; first evaluation: minimal $=42.5 \%$, mild $=27.1 \%$; moderate $=19.5 \%$, and severe $=10.8 \%$. IQR, interquartile range.

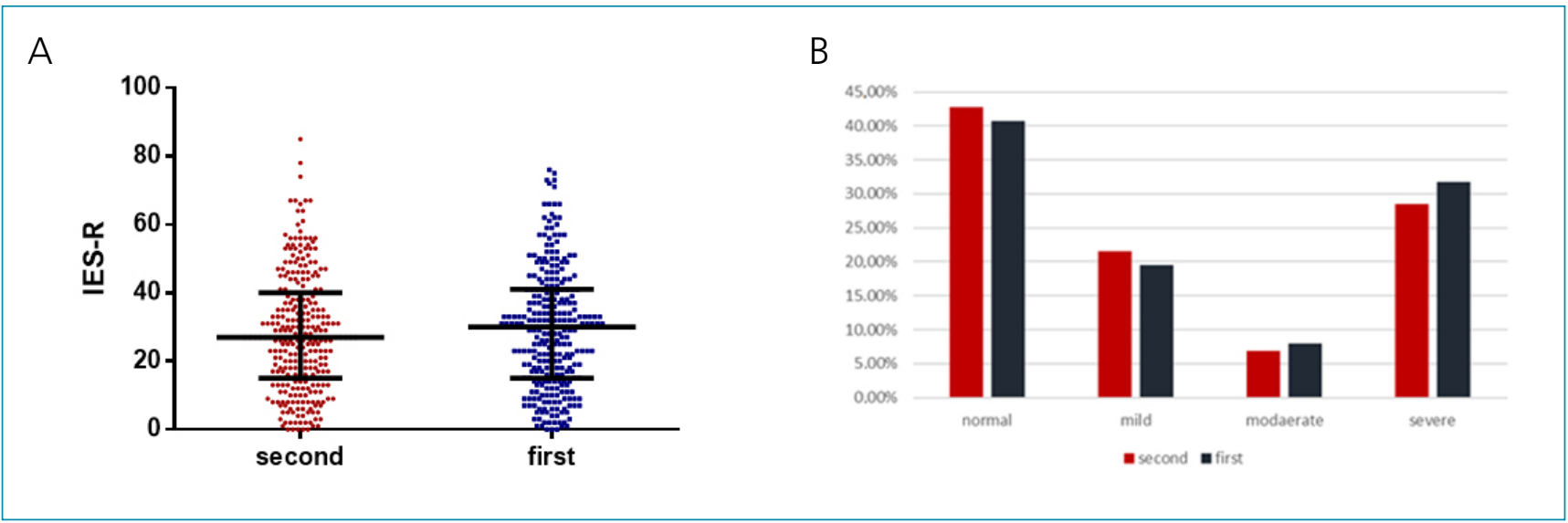

Figure 2. Comparison of IES-R (Impact of Event Scale-Revised) between the two evaluations. (A) Numeric values $p=0.0 y$; second evaluation: median values of 27.0 (IQR 15.0-40.0); first evaluation: median values of 30.0 (IQR 15.0-41.0). (B) Categorical values: $\mathrm{p}=0.76$; second evaluation: normal $=42.8 \%$, mild $=21.6 \%$, moderate $=6.9 \%$, and severe $=28.5 \%$; first evaluation: normal $=40.7 \%$, mild $=19.5 \%$, moderate $=8.0 \%$, and severe $=31.7 \%$. IQR, interquartile range .

-1.0 (IQR from -5.0 to +2.0 ); the median variation of IES-R (delta IES-R) was -1.0 (IQR -8.0 to +6.0).

The delta Beck and delta IES-R did not change according to gender ( $\mathrm{p}=0.10$ and $\mathrm{p}=0.11$, respectively) and according to keeping quarantine at second visit $(\mathrm{p}=0.63$ for Beck inventory and $p=0.28$ for IES-R).

The IES-R did not change if the individual was working/ studying in the health area $(\mathrm{p}=0.19)$ but the Beck inventory had a significant decrease as follows: median value of 0 (IQR -2.7 to +4.0$)$ for those who were not working in the health area and -2.0 (IQR -7.0 to +2.0$)$ for those who were working in the health area, with $\mathrm{p}<0.0001$.
A small and positive correlation was found between age and delta Beck (rho $=0.14 ; 95 \%$ CI $0.02-0.25 ; p=0.01$ ) but not with delta IES-R (rho $=0.01 ; 95 \%$ CI -0.10 to $+0.12 ; \mathrm{p}=0.83$ ).

\section{DISCUSSION}

Our results have shown that almost half of the study sample had some degree of anxiety and suffered some amount of psychological impact as measured by IES-R with the COVID-19 pandemic. The results were worse in females, in those who were working/studying in the health area, and in those who were keeping quarantine. We also noted that Beck inventory and IES-R score had a negative correlation with age, being worse in 
younger people. Interestingly, the second evaluation which was performed 8 weeks later showed some degree of improvement. Gender and keeping quarantine did not influence the observed changes over time but the BAI improved in those with higher age and those who were working/studying in the health area.

Finding worse results for female individuals was also observed in other surveys done in Germany, China, and Iran 2,5,7. In these three studies, similar to our results, younger people had more anxiety, despite the results unknown for the severity of COVID-19 infection in older people with co-morbidities ${ }^{1}$.

Females show more anxiety in general ${ }^{11}$. Although it is difficult to separate social and environmental factors from hormonal factors that can contribute to this preference, there are some suggestions that gonadal hormones may influence mental health. Animal studies in mice at puberty suggest that testicular hormones may have anxiolytic effects in males which are not consistent with the role of ovarian hormones in increasing anxiety ${ }^{20}$.

Individuals who were linked to the area of health showed worse outcomes. A better knowledge of the infectious process spreading and of the possible consequences of the infection may lead to a more realistic point of view, contributing to higher levels of anxiety. Fear of contamination in the work/study area may also have contributed to these results. However, it was this group of individuals who showed some improvement in the anxiety levels in the second evaluation. Growing information about the pathophysiology and treatment of the disease overcoming the initial contradictory information overload may have contributed to this improvement. So, a positive emotion regulation may have occurred. However, it is important to note that only the BAI improved but not the IES-R. IES-R is an instrument with better discriminating validity and diagnostic utility for PTSD that can be considered a subtype of anxiety ${ }^{21}$.

A Chinese study noticed that there was a decrease in the IES-R score after 4 weeks when compared with the first assessment that was carried out during the beginning of the COVID-19 pandemic, which is different from the results obtained in this study ${ }^{22}$.
This study has the following limitations. It is an Internet survey so that it reaches only people with computer resources and may not include individuals with lower income and people in rural areas, ${ }^{23}$ creating a selection bias. It also has a limited period between the two interviews. However, it shows the big impact of the COVID-19 pandemic on the mental status of the study sample. This aspect should not be neglected as it may lead to further social and economic losses. Measurements to reduce this impact are accepted although it is not easily taken. Physical distancing does not mean emotional distancing and the use of all communication resources should be stimulated to reduce the feeling of loneliness and helplessness imposed by quarantine. Exercises and meditation are nonpharmacological interventions known to have some positive effects on the anxiety symptoms ${ }^{24}$ and may be of help in selected individuals. Telemedicine through telephone or computer networks has offered an alternative option for doctor-patient communication $^{25}$, including online psychotherapy that has been shown a cost-effective way to reduce psychological symptoms ${ }^{26}$. Internet cognitive behavioral therapy has already been successfully used in other conditions such as insomnia ${ }^{27}$, chronic pain situations such as rheumatoid arthritis ${ }^{26}$, and in the treatment of comorbid depressive symptoms among individuals who have PTSD ${ }^{27}$.

\section{CONCLUSIONS}

A high prevalence of anxiety in the study sample was found. Females, younger people, and people who were working/studying in the area of health are associated with worse results. The level of anxiety showed a small reduction over time.

\section{AUTHORS" CONTRIBUTIONS}

ACMS, AZM, PZM, RAW, and RNYK: Conceptualization, Data Curation, Methodology, Writing - Original Draft. TLS: Data Curation, Investigation, Writing - Review and Editing.

\section{REFERENCES}

1. Singhal T. A review of coronavirus disease-2019 (COVID-19). Indian J Pediatr. 2020;87(4):281-6. https://doi.org/10.1007/ s12098-020-03263-6

2. Wang C, Pan R, Wan X, Tan Y, Xu L, Ho C et al. Immediate psychological responses and associated factors during the initial stage of the 2019 Coronavirus Disease (COVID-19) epidemic among the general population in China. Int J Environ Res Publ Health. 2020;17(5):E1729. https://doi.org/10.3390/ ijerph17051729
3. Qiu J, Shen B, Zhao M, Wang Z, Xie B, Xu Y. A nationwide survey of psychological distress among Chinese people in the COVID-19 epidemic: implications and policy recommendations. Gen Psychiatr. 2020;33(2):e100213. https://doi.org/10.1136/ gpsych-2020-100213

4. Sher L. COVID-19, anxiety, sleep disturbances and suicide. Sleep Med. 2020;70:124. https://doi.org/10.1016/j. sleep.2020.04.019 
5. Bäuerle $A$, Teufel $M$, Musche $V$, Weismüller B, Kohler $H$, Hetkamp $M$, et al. Increased generalized anxiety, depression and distress during the COVID-19 pandemic: a cross-sectional study in Germany. J Public Health (Oxf). 2020;42(4):672-8. https://doi.org/10.1093/pubmed/fdaa106

6. Liu CH, Zhang E, Wong GTF, Hyun S, Hahm HC. Factors associated with depression, anxiety, and PTSD symptomatology during the COVID-19 pandemic: Clinical implications for U.S. young adult mental health. Psychiatry Res. 2020;290:113172. https://doi.org/10.1016/j.psychres.2020.113172

7. Moghanibashi-Mansourieh A. Assessing the anxiety level of Iranian general population during COVID-19 outbreak. Asian J Psychiatr. 2020;51:102076. https://doi.org/10.1016/j. ajp.2020.102076

8. Xiong J, Lipsitz O, Nasri F, Lui LMW, Gill H, Phan L, et al. Impact of COVID-19 pandemic on mental health in the general population: A systematic review. J Affect Disord. 2020;277:55-64. https://doi.org/10.1016/j.jad.2020.08.001

9. Wang C, Chudzicka-Czupała A, Grabowski D, Pan R, Adamus K, Wan X, et al. The association between physical and mental health and face mask use during the COVID-19 pandemic: a comparison of two countries with different views and practices. Front Psychiatry. 2020;11:569981. https://doi.org/10.3389/ fpsyt.2020.569981

10. Brooks SK, Webster RK, Smith LE, Woodland L, Wessely S, Greenberg N, et al. The psychological impact of quarantine and how to reduce it: rapid review of the evidence. Lancet. 2020;395(10227):912-20. https://doi.org/10.1016/S01406736(20)30460-8

11. Chew NWS, Ngiam JN, Tan BY, Tham S-M, Tan CYS, Jing M, et al. Asian-Pacific perspective on the psychological wellbeing of healthcare workers during the evolution of the COVID-19 pandemic. BJPsych Open. 2020;6(6):e116. https:// doi.org/10.1192/bjo.2020.98

12. Chew NWS, Lee GKH, Tan BYQ, Jingc $M$, Gohcet $Y$, Ngiam NJH, et al. A multinational, multicentre study on the psychological outcomes and associated physical symptoms amongst healthcare workers during COVID-19 outbreak. Brain Behav Immun. 2020;88:559-65. https://doi.org/10.1016/j.bbi.2020.04.049

13. Dang AK, Le XTT, Le HT, Tran BX, Tranh TTT, Phan HTB et al. Evidence of COVID-19 Impacts on Occupations During the First Vietnamese National Lockdown. Ann Glob Health. 2020;86(1):112. https://doi.org/10.5334/aogh.2976

14. Tan W, Hao F, McIntyre RS, Jiangc,L, XiaojiangJ, Zhangc L, et al. Is returning to work during the COVID-19 pandemic stressful? A study on immediate mental health status and psychoneuroimmunity prevention measures of Chinese workforce. Brain Behav Immun. 2020;87:84-92. https://doi. org/10.1016/j.bbi.2020.04.055

15. Quintão S, Delgadob RA, Prieto G. Validity study of the Beck Anxiety Inventory (Portuguese version) by the Rasch Rating Scale Model. Psicol Reflex Crit. 2013;26(2):305-10. https:// doi.org/10.1590/S0102-79722013000200010
16. Caiuby AVS, Lacerda SS, Quintana MI, Torii TS, Andreoli SB. Cross-cultural adaptation of the Brazilian version of the Impact of Events Scale-Revised (IES-R). Cad Saude Publica. 2012;28(3):597603. https://doi.org/10.1590/s0102-311×2012000300019

17. Le XTT, Dang AK, Toweh J, Nguyen QN, Le HT, Do TTT, et al. Evaluating the psychological impacts related to COVID-19 of Vietnamese people under the first nationwide partial lockdown in Vietnam. Front Psychiatry. 2020;11:824. https:// doi.org/10.3389/fpsyt.2020.00824

18. Tee ML, Tee CA, Anlacan JP Aligam KJG, Reyes PWC, Kuruchittham $V$, et al. Psychological impact of COVID-19 pandemic in the Philippines. J Affect Disord. 2020;277:37991. https://doi.org/10.1016/j.jad.2020.08.043

19. Hao F, Tan W, Jiang L, Zhang L, Zhao X, Zou Y, et al. Do psychiatric patients experience more psychiatric symptoms during COVID-19 pandemic and lockdown? A Case-control study with service and research implications for immunopsychiatry. Brain Behav Immun. 2020;87:100-6. https://doi.org/10.1016/j. bbi.2020.04.069

20. Boivin JR, Piekarski DJ, Wahlerg JK, Wilbrecht L. Age, sex, and gonadal hormones differently influence anxietyand depression-related behavior during puberty in mice. Psychoneuroendocrinology. 2017;85:78-87. https://doi. org/10.1016/j.psyneuen.2017.08.009

21. Dean E. Anxiety. Nurs Stand. 2016;30(46):15. https://doi. org/10.7748/ns.30.46.15.s17

22. Wang C, Pan R, Wan X, Tan Y, Xu L, Mclntyre RS, et al. A longitudinal study on the mental health of general population during the COVID-19 epidemic in China. Brain Behav Immun. 2020;87:40-8. https://doi.org/10.1016/j.bbi.2020.04.028

23. Tran BX, Phan HT, Nguyen TPT, Hoang MT, Vu GT, Thi Lei H, et al. Reaching further by village health collaborators: The informal health taskforce of Vietnam for COVID-19 responses. J Glob Health. 2020;10(1):010354. https://doi.org/10.7189/ jogh.10.010354

24. Saeed SA, Cunningham K, Bloch RM. Depression and anxiety disorders: Benefits of exercise, yoga, and meditation. Am Fam Physician. 2019;99(10):620-7. PMID: 31083878

25. Tran BX, Hoang MT, Vo LH et al Telemedicine in the COVID-19 Pandemic: Motivations for Integrated, Interconnected, and Community-Based Health Delivery in Resource-Scarce Settings? Front Psychiatry. 2020;11:564452. https://doi.org/10.3389/ fpsyt.2020.564452

26. Zhang MW, HO RC. Moodle: The cost-effective solution for internet cognitive behavioral therapy (I-CBT) interventions. Technol Health Care. 2017;25(1):163-5. https://doi.org/10.3233/ THC-161261

27. Soh HL, Ho RC, HO CS, Tam WW. Efficacy of digital cognitive behavioral therapy for insomnia: a meta-analysis of randomized controlled trials. Sleep Med. 2020;75:315-25. https://doi. org/10.1016/j.sleep.2020.08.020 